\title{
Philosophiques
}

\section{Formes de complexion, types de connexion.: Remarques sur la dualité descriptive et génétique de la notion de Gestalt chez Mach, Ehrenfels et Meinong}

\section{Jean-Maurice Monnoyer}

Volume 26, numéro 2, automne 1999

La critique de la raison en Europe centrale

URI : https://id.erudit.org/iderudit/004957ar

DOI : https://doi.org/10.7202/004957ar

Aller au sommaire du numéro

Éditeur(s)

Société de philosophie du Québec

ISSN

0316-2923 (imprimé)

1492-1391 (numérique)

Découvrir la revue

Citer cet article

Monnoyer, J.-M. (1999). Formes de complexion, types de connexion.:

Remarques sur la dualité descriptive et génétique de la notion de Gestalt chez Mach, Ehrenfels et Meinong. Philosophiques, 26(2), 245-261.

https://doi.org/10.7202/004957ar
Résumé de l'article

Le but de cet article est de confronter trois acceptions du terme Gestalt, concept et entité qui a joué en Europe un rôle considérable dans l'émergence de la phénoménologie et de la psychologie descriptive entre 1890 et 1930, avant que les représentants de l'école berlinoise n'émigrent aux États-Unis. On confronte ici le sens donné à l'appréhension de la Gestalt, d'abord chez E. Mach, puis chez le fondateur de ce courant de pensée, C. von Ehrenfels, et enfin chez Meinong, dont Ehrenfels a été l'élève. Le problème central de la relation de complexité et de son "fondement » ontologique dans le domaine particulier de l' écoute musicale est abordé par ces trois auteurs. On explique pourquoi leurs divergences ont ensuite conduit à remplacer, par une conception psycho-génétique (celle de Koffka et de Köhler), ce qui se présentait à l’origine comme une espèce nouvelle de relation que la " forme " entretient à l'égard de ses éléments constitutifs, sans jamais dépendre d'eux par une explication causale. 


\title{
Formes de complexion, types de connexion.
}

\author{
Remarques sur la dualité descriptive et \\ génétique de la notion de Gestalt chez \\ Mach, Ehrenfels et Meinong \\ JEAN-MAURICE MONNOYER \\ Université Pierre Mendès France, Grenoble II \\ Jean-Maurice.Monnoyer@upmf-grenoble.fr
}

\begin{abstract}
RÉSUMÉ. - Le but de cet article est de confronter trois acceptions du terme Gestalt, concept et entité qui a joué en Europe un rôle considérable dans l'émergence de la phénoménologie et de la psychologie descriptive entre 1890 et 1930, avant que les représentants de l'école berlinoise n'émigrent aux États-Unis. On confronte ici le sens donné à l'appréhension de la Gestalt, d'abord chez E. Mach, puis chez le fondateur de ce courant de pensée, $C$. von Ehrenfels, et enfin chez Meinong, dont Ehrenfels a été l'élève. Le problème central de la relation de complexité et de son «fondement » ontologique dans le domaine particulier de l'écoute musicale est abordé par ces trois auteurs. On explique pourquoi leurs divergences ont ensuite conduit à remplacer, par une conception psycho-génétique (celle de Koffka et de Köhler), ce qui se présentait à l'origine comme une espèce nouvelle de relation que la « forme » entretient à l'égard de ses éléments constitutifs, sans jamais dépendre d'eux par une explication causale.
\end{abstract}

\begin{abstract}
The aim of this article is to contrast three readings of the term Gestalt, a concept and an entity which has had a considerable role in the emergence of phenomenology and descriptive psychology, between 1890 and 1930, before the members of the Berlin school go to the U.S.A. We contrast the sense given to the apprehension of the Gestalt by Ernst Mach with the one it takes in the works of $\mathrm{C}$. von Ehrenfels, the founder of this school of thought, and then with its sense in the works of Ehrenfels' master, Meinong. The central problem of the complexity relation and of its ontological "foundation" is discussed by these three thinkers, with respect to the special case of musical listening. We explain why their diverging views led to replace what at the beginning was a new kind of relation which the "form" could entertain with its constitutive elements without causally depending on them, by a psycho-genetic conception (that of Koffka and Kölher).
\end{abstract}

Dans son édition des Principles of G estalt Psychology, Koffka évoque le genre d' ' isomorphisme » qu'on peut établir entre les processus conscients et les processus physiologiques. II mentionne à ce propos que les travaux de Wertheimer et de Köhler, publiés dans les années 20 , sont la reprise sophistiquée et détaillée d'un thème plus ancien développé simultanément par $M$ ach et $H$ ering soixante-dix ans plus tôt. Ce thème admet, selon eux, un principe psychologique fondamental d'après lequel un tel isomorphisme devrait être défini de manière «dynamique », c'est-à-dire en fonction de l'environnement ou du comportement intentionnel du sujet, mais non pas du tout causalement. La notion d'une dépendance non causale de la G estalt est décisive. Elle nous autorise à justifier le dynamisme en question dans le 
domaine de l'expérience directe. D'après K offka cependant, on ne doit pas exclure qu'il puisse y avoir des stimuli corrélatifs de la G estalt : il affirme seulement que l'expérience directe que nous en faisons est immédiatement organisée. Et puisque les stimulations sensorielles ne sont pas des « causes » extérieures au processus perceptif, de tels stimuli ne se distinguent pas «fonctionnellement », d'après Koffka toujours, des stimuli « primaires » ${ }^{1}$. On doit supposer donc (dans l'hypothèse où il y aurait une similarité objective entre les processus conscients et les processus physiologiques capable d'expliquer cette « dépendance ») qu'il faut aussi qu'une différence subsiste entre le fondement de son apparition et la reconnaissance de la G estalt. Le «parallélisme psychophysique » de M ach peut-il en rendre compte? Koffka écrit à cet endroit :

Le principe était si peu connu que $K$ öhler, qui se réfère à $H$ ering et $M$ üller, ne mentionne pas $M$ ach dans cette filiation. J'ai trouvé le passage en question dans $\mathrm{M}$ ach tout à fait par hasard et à ma grande surprise. $\mathrm{N}$ ous n'avons pas besoin de chercher très loin pour trouver la raison de cette apparente injustice historique. $M$ ach était un excellent physiologiste, qui a entrevu beaucoup des principaux problèmes de la psychologie, ceux qu'une génération entière de psychologues après lui échouèrent à comprendre; mais en même temps, sa philosophie ne permettait pas de donner des solutions fructueuses aux problèmes qu'il avait soulevés. ${ }^{2}$

On ne reviendra pas sur la relative désinvolture avec laquelle Ernst $M$ ach introduit le concept et le mot de $G$ estalt, contrastant avec la grande finesse des aperçus que L'A nalyse des sensations recèle à ce sujet. $N$ otons que la position même de K offka, indépendamment du catalogue d'observations qu'il propose, privilégie l'explication physiologique et la distribution des stimuli tout en restant fort silencieuse quant aux raisons philosophiques qui nous pousseraient à admettre le dynamisme non causal constitutif de cette entité nouvelle. $M$ ach est demeuré très prudent, et non sans motif, à l'égard de l'idée d'une causalité efficace. Sa conception n'est pas très différente de celle de $\mathrm{H}$ ume, et confine à l'abstinence métaphysique en ce domaine. Aux yeux du lecteur moderne, cette économie sémantique va de pair avec la résistance que nous éprouvons encore à isoler la figure distinctive que recouvre la $\mathrm{G}$ estalt. En d'autres termes, ce qui la produit ne nous dit rien, semble-t-il, de

1. Le départ de cette distinction se trouve dans I'opposition des élèves de Stumpf aux découvertes del'école de Graz. A insi K offka s'oppose-t-il de front à Benussi en 1915, dans « Zur Grundlegung der Wahrnehmungpsychologie. Eine Auseinandersetzung mit V. Benussi », Z eitschr. f. Psychol., 1915, 73, p. 11-90, repris dans Ellis, W. D., dir., A Sourcebook of G estalt Psychology, 1938, p. 371-378. Pour Benussi, la G estalt est une «production » qui n'a pas de stimuli, et se constitue comme un objet « idéal » meinongien. La relation constitutive de la G estalt est appelée « R ealrelation » : elle est surimposée à des éléments physiques. À I'inverse, pour Koffka, les sensations primaires n'apparaissent que comme un produit de l'analyse et dans les conditions particulières où se décompose le « tout » structurant de la G estalt.

2. Koffka, Kurt, Principles of G estalt Psychology, R outledge and Kegan, 1935, p. 63. 
la façon dont elle est appréhendée. À ce niveau du débat, pourquoi faudraitil offrir une clarification supplémentaire? Kevin M ulligan et Barry Smith ont présenté déjà une mise au point précise et sondé à la verticale les strates phénoménologiques du problème amplement documenté de la $\mathrm{G}$ estalt ${ }^{3}$. Pour écarter le risque de proposer ici une nouvelle complication de la question, il convient de se limiter à la dimension philosophique du problème. Le passage d'une notion issue de la psychologie génétiqueà une notion relevant du point de vue descriptiviste adopté par les Viennois de la seconde génération demeure, en effet, difficileà tracer. $\mathrm{N}$ otre but est seulement de parvenir à spé cifier la nature des complexes dont la G estalt est formée, en les distinguant des types de connexions qu'on invoque pour les définir.

\section{1}

A fin d'entrer de plain-pied dans la genèse du concept, on pourrait par exemple délimiter un cadre historique volontairement restreint. $N$ otre choix s'est porté sur une période de temps limitée, qui comprend : 1) l'introduction de cette entité toujours assez mal connue, telle qu'elle est présentée par $M$ ach dans son Analyse der E mpfindungen en 1886, tout en sachant qu'elle n'est pas exploitée par lui directement ; 2) I'article novateur d'Ehrenfels, Über G estaltqualitäten, paru en 1890 ; et 3) la recension que fit $M$ einong de ce même article en 1891, Zur Psychologie der Komplexionen und Relationen. Ces trois références, groupées sur un arc de cinq années, permettent d'attester l'origine exclusivement autrichienne d'une telle discussion, qui se déploie d'abord dans l'École de Graz, avant d'être amplifiée par les cercles intellectuels de Prague et de Berlin4. Plutôt que d'opérer une comparaison systématique, nous n'évoquerons que quelques traits saillants du débat naissant.

On admet d'ordinaire comme une insuffisance de la part de $M$ ach la difficulté à incorporer sa description des complexes (ou encore des « complexions », comme diront plus tard $M$ einong et ses élèves) avec le cadre théorique de l'élémentarisme. Tel est bien le reproche implicite à l'hommage ambigu que lui rend Koffka. $M$ ach distingue en réalité, parce qu'il les « analyse » séparément :

1) les types d'appréhension simples attachés aux éléments singuliers que sont les sensations ( $\mathrm{G}$ rundempfindungen, ou Sinnesempfindungen) ${ }^{5}$.

3. Mulligan, K. et B.Smith, «M ach and Ehrenfels: The Foundations of Gestalt Theory », dans Smith, B., dir., Foundations of G estalt Theory, M unich, Philosophia, 1988, p. 124-157.

4. N ous avons mis de côté les répercussions de cette discussion dans I'œuvre de H usserl, qui, dans sa Philosophie de l'A rithmétique (1891), intitule « moment figural » la découverte d'Ehrenfels afin d'éviter des confusions fréquentes.

5. Ernst Mach, Die Analyse der Empfindungen, Fischer, Iena (1886-1911); réédition : W issenschaftliche Buchgesellschaft, Darmstadt, 1991 ; traduction française : L'A nalyse des sensations, Éditions J. Chambon, 1996. Ces éléments singuliers sont dépendants 
2) les modes de recognition d'une qualité « isomorphe » (d'une ressemblance) entre différents complexes, dans lesquels les mêmes éléments de départ sont combinés différemment, parce qu'appréhendés autrement.

Contrairement à ce que pense $K$ offka, la relation de similariténe repose pas (comme en 1) sur une identification psychosomatique qui se ferait dans la seule appréhension des phénomènes perceptuels. $M$ ach construit deux séries séparées de rapport au monde phénoménal, en invoquant un parallélisme strict. Ainsi pouvons-nous seulement suspecter qu'une nécessité d'un autre genre que la nécessité physique les met provisoirement en parallèle. La variété des connexions possibles laisse aussi penser que nous devons faire l'économie d'une nécessité univoque. Prenons le cas le plus disputé : celui des Tonempfindungen. D'un côté, la perception d'une note isolée, quand on fixe sa hauteur absolue, suppose une appréhension spécifique de son caractère (de l'énergie qui la produit) : ce caractère distinctif ne « dépend » ni de l'intervalle où elle est située dans la succession de deux ou trois autres notes, ni de sa composition harmonique dans le spectre sonore. De l'autre, la perception d'un complexe (d'un ensemble défini de notes) induit la caractérisation d'unequalité qui n'est plus psychologiquement « simple». Comptetenu de cette distinction préalable, nous devrons admettre qu'à cet égard, Ehrenfels « dira » effectivement quelque chose de plus, sans ajouter quelque chose de nouveau à l'exposition de L'A nalyse.

Pourtant, qui voudrait défendre $M$ ach se limitera à observer que cette perception de la G estalt ne suppose pas chez lui une perception « distincte » de la relation, comme celle qu'on fait, par exemple, de la répétition d'un rapport harmonique identique entre deux tierces. II n'y a pas de G estalt qualitativement remarquable, au sens consacré du mot, dans un tel cas. Sans doute le problème de la perception des relations est-il un problème très aigu, auquel s'est confronté $M$ einong et par la suite Russell dans sa Theory of Knowledge ${ }^{6}: M$ ach se contente d'avancer que cette relation existant entreles éléments du complexe « mélodique » demeure « sous-jacente » au complexe des sensations. Cela implique que la recognition de la mélodie, qui certes ne produit pas physiquement cette relation, ne « dépend » pas non plus d'un calcul qui se ferait dans la sphère intelligible (c'est la conviction de Stumpf, que conteste vigoureusement la troisième édition de L'A nalyse, ch. XIII, $\S 18)$. La non-dépendance causale se double d'une irréductibilité cognitive dela relation. 0 n le mesure très clairement dans ce passage, où les conditions des processus nerveux s'opposent à l'assimilation cognitive des mêmes processus :

de l'appréhension nerveuse qui les différencie en sensations de temps, d'espace, de couleur, de son, etc. À leur sujet, $\mathrm{M}$ ach fait une hypothèse qui concerne le processus nerveux physiologiquement déterminable, auquel devrait correspondre la saisie « psychologique » de ce qui est simple.

6. Russell, Theory of Knowledge, 1913, ch. VII. 
Si je vois identiques deux $G$ estalten de couleurs différentes, je chercherai à côté des sensations de couleurs différentes certaines sensations d'espace identiques, ainsi que des processus nerveux identiques qui leur correspondent. Si ces deux Gestalten se ressemblent (c'est-à-dire reposent en partie sur les sensations d'espace identiques), les processus nerveux correspondants contiennent en partie les mêmes composantes. Si deux mélodies différentes ont un rythme identique, il existe dans les deux cas, à côté des sensations de son différentes, une même sensation de temps avec les mêmes processus correspondants. Q ue deux mélodies soient identiques dans des registres différents, les sensations de son ainsi que leurs conditions physiologiques ont des composantes identiques, malgré la différence de hauteur. ${ }^{7}$

Les deux dernières éventualités correspondent aux Z eitgestalten et aux Tongestalten sur lesquelles il revient dans les chapitres ultérieurs ${ }^{8}$. S'agissant de la dernière forme, il est notable que la différence de hauteur du son soit inopérante : les sensations caractéristiques sont préservées, ainsi que leurs conditions nerveuses, tandis que l'identification singulière des notes n'est plus constitutive de la G estalt. Les « sons isolés » sont fixés dans l'espace unidimensionnel del'échelle sonore ${ }^{9}$; ils sont le fruit d'une analyse réductive menée par H elmholtz : elle ne conditionne aucunement la sensation caractéristique de l'intervall ${ }^{10}$. Pour résumer ce point, il semble que $M$ ach ait marqué une différence entre le mode de représentation particulier du complexe mélodique et la structure distributive des sons dépendant « fonctionnellement » de l'appareil récepteur de l'ouïe, mais aussi qu'il soit demeuré incapable de la définir. Comment accorder ces deux dimensions de l'écoute, I'une quantitative ou physiologique (un rapport de fréquences), l'autre qualitativement définie?

Le combat de $M$ ach contre le dualisme récuse bien sûr cette opposition. II exige en effet qu'une sorte de nécessité soit au moins postulée au bénéfice de cette dépendance dont nous parlions, puisque sont combinées une appré hension primaire (variable) et une recognition qualitative dela ressemblance. Sont-elles dépendantes I'une de l'autre, parce qu'elles sont conjuguées I'une et l'autre? Voulant résoudre la question de savoir comment pourrait s'effectuer cette connexion, dont le type est déclaré par lui « logique», $M$ ach en appelle à un processus organique autonome, surajouté à la perception des unités singulières, lequel processus (qui, il est vrai, symbolise seulement cette connexion) est activé par des sensations musculaires locales, liées par exemple aux mouvements oculomoteurs ou aux vibrations ciliaires de la membrane auditive. Les mêmes muscles sont innervés par les globes de l'œil quand nous reconnaissons l'identité composite d'une forme spatiale orientée de manière homologue dans le champ visuel; et les mêmes fibres nerveuses

7. $M$ ach, D ie A nalyse der Empfindungen, p. 52 (trad. fr. p. 61).

8. Ibid., ch. XII et XIII.

9. Ibid., ch XIII, § 10 .

10. Ibid., p 234 (trad. fr. p 252) 
sont excitées par le mouvement des cils auditifs et de leurs terminaisons nerveuses, quand nous reconnaissons la complexité d'une mélodie identique, transposée dans un autre registre. Ces mouvements, mécaniquement déterminables, nous procurent des sensations musculaires qui ne sont pourtant pas appréhendées pour elles-mêmes (souvent, elles ne sont pas conscientes) : elles ne sont pas « distinguées » au sens strict, ni entre elles nécessairement connexes. $K$. M ulligan et $B$. Smith ${ }^{11}$ constatent pour leur part que ce phénomène additionnel ne saurait expliquer la perception instantanée d'un complexe où les sensations sont « appréhendées ensemble » : ils soulignent une inadéquation foncière dans le parallélisme invoqué entre le fondement sensori-moteur et le statut ontologique et psychologique de la $\mathrm{G}$ estalt. Ehrenfels, à la différence de $M$ ach, aurait évoqué le premier la possibilité $d^{\prime}$ '« une dépendance mutuelle entre la qualité de G estalt et son fondement, non pas simplement au sens logique mais aussi dans son sens ontologique ${ }^{12}$.

Cette inadéquation peut disparaître en partie, malgré l'impression de flou terminologique qui demeure très réelle, si l'on parvient à détecter quelques propriétés différenciantes de la sensation, que l'auteur a énoncées sans les ordonner dans son maître ouvrage. D'abord, sel on ce qu'il appelle en effet principe de « différenciation suffisante », une sensation élémentaire peut être étudiée psychologiquement comme un en soi qualitatif, dans la tradition de Johannes $M$ üller ${ }^{13}$. Les sensations musculaires élémentaires ne sont pas du tout de cette nature. De tels mouvements ne fournissent en réalité qu'une « résonance corporelle », d'après l'expression qu'il emploie dans ses Bemerküngen zur $L$ ehre vom räumlichen Sehen, en $1865^{14}$. C'est pourtant par l'occasion de ces sensations musculaires que s'opère la connexion requise, sans que $M$ ach se prononce plus avant sur la nature de cette connexion. La contrainte structurale de la G estalt, de même que son détachement par rapport à d'autres, ne sont pas évoqués par lui. L'idée d'une succession dans cette articulation d'éléments d'abord isolés, puis associés (avant même donc

11. M ulligan, $K$ et $B$. Smith, " $M$ ach and Ehrenfels: The Foundations of Gestalt Theory », p. 128.

12. Ibid., p. 132.

13. M ach, D ie A nalyse der Empfindungen, Ch IV, § 2, p 49 (trad. fr. p 58), « la sensation peut désormais être analysée en soi, immédiatement, autrement dit psychologiquement [...]. $\mathrm{Ou}$ bien on pourrait se mettre à la recherche des processus physiques [physiologiques] qui lui sont coordonnés [...] ou bien enfin on peut s'intéresser à la connexion [Z usammenhang] entre ce qui est psychologiquement observable et les processus physiques (physiologiques) corrélatifs des premiers (c'est ce qui nous conduira le plus loin) ». Dans ce passage, le genre de connexion n'est pas rapporté à ce qui relie entre elles des sensations analytiquement séparées, selon la méthode de réduction des physiologistes. L'analyse psychologique de ce qui est observable, et supposé simple, rencontre parfois des exceptions : si nous décomposons (Z erlegung) une sensation sonore quelconque, nous ne pouvons réduire à des «éléments » ses parties mutuellement indépendantes (einander unabhändige Bestandteile), comme l'intensité et la hauteur du son. $M$ ais il est possible de le faire pour la sensation de provenance du son dans l'espace.

14. Cf. M ulligan, K et B. Smith, « M ach and Ehrenfels: The Foundations of Gestalt Theory », p. 127. 
que se produise la recognition d'un complexe), ne se pose pas non plus dans les termes de L'A nalyse des sensations en référence à l' « unité » présumée de la $G$ estalt. Pour $M$ ach, le rythme et lui seul impliquela succession. Plus exactement, la succession est « neutre » eu égard au fait que nous organisons les sons en série (Tonfolge), alors que ce sera le thème prioritaire développé par Ehrenfels. Enfin, l'expression de « résonance corporelle » peut aussi être prise à la lettre, car rien n'interdit de penser que la polémique qui l'opposa à Stumpf sur l'identification psychosomatique de la résonance acoustique n'ait pas provoqué chez $M$ ach le désir de fonder, dans ce « complexe d'éléments » qu'est notre corps, une réponse sensorielle à la disparité aléatoire des stimuli auditifs.

Il importe désormais de tenter d'identifier plusieurs types de connexions, si nous voulons fournir une évaluation correcte de sa doctrine. Une lecture bienveillante relèverait chez $M$ ach :

a) une connexion nerveuse (qui est en fait une correspondance indispensable au parallélisme entre des états psychologiques et des états énergétiques) ;

b) une connexion sensori-musculaire (qui assure la dépendance fonctionnelle et non causale des «qualités » par rapport aux mouvements mécaniques) ;

c) une connexion logique - mais aussi psychologique - , celle-là propre au complexe de sensations qui forme la $\mathrm{G}$ estalt, et que la deuxième symbolise ${ }^{15}$.

Les connexions $a$ ) et $b$ ) sont hiérarchiquement plus proches. $M$ ais toutes les trois se tiennent dans une relation d'interdépendance qu'il est difficile de préciser mieux. D ans le troisième cas, $M$ ach a défendu la thèse que ce genre de connexion est immédiat - qu'il n'est jamais « intellectuel » - , et, par voie de conséquence, que les abstractions que nous formons ensuite dans la représentation sont fondées, en dernière instance, sur un contact dynamique et intradermal, qui se réalise toujours à partir de la rencontre du monde extérieur, bien qu'essentiellement par « habitude ». Les données psychologiques $\alpha, \beta, \gamma$, etc. se coordonnent avec des éléments organiques $K, L, M$, etc. $O n$ comprend mieux ce que K offka ne fit qu'entrevoir dans son évaluation rétrospective. Les objets résonnants et les qualités mélodiques de la G estalt sont les deux maillons terminaux d'une même chaîne. Ce contact, s'il était permis de le dire ainsi dans les catégories de $\mathrm{M}$ ach - et d'une manière presque abusive dans le langage - , se ferait « à l'intérieur » du monde extérieur, un monde où s'établit, dira excellemment $M$ einong pour s'opposer à la définition psychologique d'Ehrenfels, la « mise en forme des choses » (G estaltung der D inge).

15. $N$ ous avons essayé de retrouver un clivage terminologique entre les trois formes: Verknüpfung, Z uzammenhang, Verbindung. $M$ ais nous ne sommes pas parvenu à établir le caractère stable de ces différences lexicales dans le texte de L'A nalyse. II semble donc que ce problème n'ait pas été déterminant dans l'optique que $M$ ach a retenue, ce que le caractère non univoque de la connexion paraît confirmer. 
Dans son essai Sur les qualités de forme, Ehrenfels récuse à la fois cette instantanéité « associative » qui caractériserait selon $\mathrm{M}$ ach la formation des représentations (Vorstellungen), et l'idée qu'un élément additionnel de nature psychosomatique, comme les sensations musculaires ou l'abaissement des cils érectiles recouvrant les ampoules du canal auriculaire, pourrait expliquer l'apparition de la G estalt.

Si nous devons, d'après Ehrenfels - que ce soit d'un seul coup dans l'espace, ou dans la durée par le soutien d'une image mémorielle - , rassembler ou collecter une pluralité quelconque, et si ce rassemblement ou cette collection sont effectivement donnés avant toute analyse des constituants, c'est que nous prêtons aux complexes une réalité cohésive assez forte : cette réalité anéantit la notion triviale d'une « somme » d'éléments (Z usammenfassung), perçue telle un agrégat amorphe. Comme l'expliquent M ulligan et Smith :

Ehrenfels reconnaît que les notes constituent en, et par elles-mêmes, un certain tout complexe, et que la qualité de Gestalt est fondée sur (plus précisément « est la qualité de ») ce tout complexe. $M$ ais la qualité elle-même n'est pas le tout embrassant les éléments comme ses parties. ${ }^{16}$

Dela même façon que $M$ ach, Ehrenfels soutient qu'aucun effort spécial n'est requis pour percevoir la qualité. Elle est appréhendée par elle-même eo ipso - , en tant que présente à la conscience en même temps que le complexe qui la fonde ([w]o und wann immer sich im Bewusstsein ein Komplex zusammen findet, w elcher « die G rundlage für eine G estaltqualität abgeben kann, ist diesselbe eo ipso und ohne unter Z utun im Bewusstsein mitgegeben ») ${ }^{17}$. En fonction de cette appréhension première, il discerne dans les textes de $M$ ach « quelque chose de nouveau, et à un certain degré d'indépendant $»^{18}$. Or, sur ce point même, E. Rausch objectera plus tard à Ehrenfels que la G estalt n'est peut-être pas autre chose qu'un « produit logique » et que, si elle n'a rien d'une sommation psychologique qui se ferait automatiquement, elle n'est pas non plus réductible à une saisie intuitive, la Konkrete Anschauung qu'il réclame ${ }^{19}$.

Peut-être faudrait-il, pour le comprendre, écarter quelques obscurités résiduelles. Si I'on adopte l'interprétation proposée par M ulligan et Smith, la dépendance unilatérale de la G estalt par rapport à ses « constituants » n'est

16. Ibid., p. 130-131.

17. Dans Weinhandl, F., dir., G estalthaftes Sehen, Darmstadt, 1974, p. 11-43 : «Quel que soit le lieu ou le moment où un complexe qui peut servir de fondement à une qualité de G estalt se trouve présent à la conscience, cette qualité est elle-même eo ipso et sans aucun effort de notre part donnée avec lui à la conscience. »

18. Ibid., p.12.

19. Rausch, Erwin, «Über Summativität und N ichtsummativität », Psychologische Forschung, 1937, 21, 209-289. 
admissible en toute rigueur que sur le plan « ontologique » et formel, et non pas sur le plan génétique, comme insisteront à ce sujet les élèves de $M$ einong20. Ils reprochent légitimement à $M$ ach d'avoir conçu une notion de " dépendance » en fin de compte peu claire, qui ruine son idée d'une nécessité logique ${ }^{21}$. Est-ce vraiment plus clair pour Ehrenfels? Chez ce dernier, le fait d'admettre une certaine spontanéité dans la reconnaissance des qualités semblables ne va pas - musicalement - de soi. II anticipe et il écarte des objections précises. Devrions-nous penser alors que l'indulgence qu'il manifeste pour les thèses de $M$ ach n'a de justification qu'esthétique? $\mathrm{N}$ on, elle a aussi d'autres conséquences. $M$ ais il est certain que la déviation à laquelle il procède en ce qui concerne les catégories musicologiques est insuffisamment soulignée. Le début de son essai est très significatif sur ce plan. Puisque chaque son (indifférencié) est lui-même erklingend, l'appréhension musicale d'une « résonance » consciente exige d'après lui une perception autre qu'acoustique. Assimilant «mélodie» et résonance psychologique, Ehrenfels repousse d'emblée la problématique compositionnelle du Klang dont tout lemonde discuteà son époque, pour n'entendre queles « qualités » qu'il veut expliquer sur de « courtes » séquences. La suite mélodique des sons $(T o n)^{22}$ suscite selon lui une impression (Eindruck) dans le souvenir, qui est indépendante du principe de la résolution harmonique et qui relève du sentiment ( $G$ efühl). Considérant «l'impression de la suite sonore » (die E indruck der Tonreihe) qui nous vient, selon lui, après coup, comme une perception à somme nulle (ce qu'il appelle curieusement l'A usklingen der M elodie ${ }^{23}$ ), il propose aussitôt de remplacer cette impression de succession par la saisie (A uffassen) de Tongestalten, lesquelles, même « non analysées », sont répercutées par le sentiment intérieur. $0 \mathrm{n}$ obtiendrait de cette manière de « pures appréhensions sonores » (rein tonal aufgefassten « Tonges-talten »)24.

Ce qu'il faut admettre, pour entendre comme lui ces « formes », est en réal itéun séquençage très particulier qui ne dépend pas justement de la structure compositionnelle de la mélodie. O n comprend volontiers, dans cette perspective, que Benussi ait ensuite objecté à ses adversaires physiologistes qu'il pouvait facilement « voir » ces $G$ estalten sur la partition. $M$ ais la définition qu'Ehrenfels fournit du « complexe représentatif » de la G estalt, en tant que Summe (pour l'opposer à une « collection » d'actes conscients), confirme qu'il s'agit d'une saisie psychologique avant tout, puisque les déterminations de temps qui appartiennent aux sons isolés sont exclusives et mutuellement distinctes (verschiedenen, sich aneinanderschliessenden Bes-

20. M ulligan, $K$ et B. Smith., « $M$ ach and Ehrenfels: The Foundations of Gestalt Theory », p. 132.

21. I bid., p. 146.

22. Et non pas des «notes».

23. Littéralement, l'« extinction sonore de la mélodie».

24. Ehrenfels, « Uber G estaltqualitäten », p. 12 et 13. 
timmheiten). Cette saisie unitaire n'est possible que grâce à l'identité de l'image mémorielle, où ces $\mathrm{G}$ estalten sont ensuite reconnues.

Le reste de l'argumentation d'Ehrenfels est mieux étudié, et il n'est pas nécessaire de s'y attarder ici. La fin de son essai permet cependant de donner une justification au remplacement terminologique qu'il réclamait de la « suite sonore » et de son organisation interne, celle qui est écrite et jouée dans une pièce de musique. D ans la succession des abstrakta de la seule notation, les « complexes » sont méconnaissables. Bien plus, Ehrenfels affaiblit progressivement, à mesure que sa notion du « complexe» se spatialise, la discrétion des éléments premiers (points ou notes). II apparaîtra à la fin de l'essai que la discrétion quantitative, notamment pour la perception des hauteurs de son et des accords, et pour celle des couleurs, est elle aussi discréditée au profit des phrases musicales et des réalisations picturales. Ehrenfels déclare entre autres choses que nous pourrions admettre l'arrière-plan d'un continuum visuel si nous pouvions descendre en dessous du seuil où sont combinées entre elles les multiples $\mathrm{G}$ estalten dont nous disposons, par exemple entre les parties colorées d'une surface. II lui semble que le pouvoir «formateur » de la représentation est sans commune mesure avec notre puissance de discrimination, qu'elle soit sonore ou visuelle.

\section{3}

Rappelons que, pour Ehrenfels, les relations de similarité indispensables à la recognition nous sont données avec un contenu positif que la conscience pose devant elle $e^{25}$. Or, c'est surtout le cas des intervalles en musique, qui ont besoin d'un temps psychologique pour être qualifiés formellement. Le même argument a été discuté par Stumpf en 1875, et l'a été de nouveau par $M$ ach en ce qui a trait aux sons partiels (les harmoniques d'un son). Cet argument se trouve repris également par Brentano, à la suite de $\mathrm{H}$ elmholtz cette fois, sur la question des micro-intervalles chromatiques, qui se situent en dessous ou à côté des sons harmoniques, à la limite du seuil de perception ${ }^{26}$. N ous avons soulignéque la recognition d'un complexe en tant que $\mathrm{G}$ estalt exige un supplément psychologique par rapport à des éléments identiques (qu'on choisisse des intervalles, des accords, ou de simples notes), qui sont intuitionnés dans la diversité du contenu représentatif. II n'en demeure pas moins pourtant qu'il nous faut conserver le principe d'une connexion ou d'une articulation relativement opératoire, sans laquelle le complexe n'existe pas.

Le point fort de la thèse d'Ehrenfels est le suivant : I'articulation interne de la mélodie, quand elle s'effectue sur la base d'une combinaison harmoni-

25. Ibid., p. 20.

26. Brentano, Franz, «Von der psychologischen Analyse der Tonqualitäten in ihre eigentlich ersten Elemente » (1905), dans U ntersuchungen zur Sinnespsychologie, M einer Verlag, 1979, p. 93-103. 
que, ne constitue pas la véritable complexitédu « complexe ». II admet comme second trait caractéristique de la G estalt sa «transposabilité ». M ais le point faible de sa conception est que cette transposition ne saurait être pour lui que de nature mélodique. Pourquoi se passer de la transposition harmonique? $\mathrm{M}$ ach en fait mention dans les pages qu'Ehrenfels a lues. Ehrenfels l'ignore pour de bonnes raisons. Le modèle de la simultanéité qui est le sien est, en priorité, d'ordre spatial ; la perception simultanée d'un complexe dans le souvenir n'est pas une audition simultanée. Plus précisément, la subsistance du complexe d'éléments n'implique pas la coexistence de la G estalt auditive et de ses éléments dans leur articulation (Z usammensein der G estaltqualität selbst und ihrer Elemente) ${ }^{27}$. N ous définirions alors une qualité de qualité, indéfiniment compliquée. Pour se restreindre à son problème, Ehrenfels est contraint (de la même façon que $M$ ach, quoique beaucoup plus délibérément que lui) de stipuler une analogie avec le monde des couleurs : les mélodies sont identifiables comme des «figures » composées de couleurs : elles doivent leur ressemblance (Ä hnlichkeit) à une ressemblance figurative, sans rapport avec la ressemblance des couleurs ${ }^{28}$. La conséquence est que les « fondements » sensoriels du complexe sont perçus comme des «lieux » de la tonalité. N ous aurons besoin, en présence de deux $\mathrm{G}$ estalten qui se ressemblent, d'une « relation analogique » (analoge Beziehung), de sorte que le contenu positif (et transposable) sera lui aussi fondé dans cette relation, en tant qu'élément représentatif : " D iese Beziehung ist nach unserere A uffassung in einem positiv Vorstellungselement, der Tongestalt, gegründet $»^{29}$. Bref, tout ce développement de sa part ne s'explique que si son but est de réfuter la saisie harmonique des intervalles.

$C$ e que nous « reconnaissons » dans le souvenir, soulignait $M$ ach, repose exclusivement sur la perception d'intervalles identiques qui se suivent (par exemple, des intervalles de quarte), parce qu'ils sont harmoniquement « combinés » entre eux ${ }^{30}$. Ehrenfels est prêt à considérer les intervalles comme des éléments du complexe. $M$ ais son intuition lui interdit de réduire la $\mathrm{G}$ estalt à un « arrangement » harmonique. $\mathrm{N}$ i la hauteur absolue des sons ni l'égalité des intervalles ne sont, dit-il, associés au souvenir de la G estalt. Les musicologues qui adoptent aujourd'hui cette théorie chromatique de la mélodie en donnent la paternitéà Ehrenfels, puisque la distance chromatique entre des chromas absolus (des « lieux » fixes) est abandonnée, et quela référence à des hauteurs définies cesse d'être effective ${ }^{31}$. Ehrenfels va beaucoup plus loin cependant, puisqu'il identifie à des $R$ aumgestalten les données précédentes de l'harmonie et du timbre (Klangfarbe), en contestant explicite-

27. Ehrenfels, « Uber Gestaltqualitäten », p. 17.

28. Ibid., p. 18.

29. Ibid., p. 20.

30. M ach, D ie A nalyse der Empfindungen, Ch. XIII, p. 13.

31. Voir DeBellis, M ark, M usic and Conceptualisation, Cambridge University Press, 1995, p. 15. 
ment $\mathrm{M}$ ach ${ }^{32}$ et en remarquant que cette analogie ne fonctionne pas en sens inverse, puisqu'il ne saurait y avoir d'harmonie simultanée des couleurs visibles 33 .

À ce stade, importe évidemment l'idée selon laquelle les accords sont des unités «fusionnelles » des couleurs du timbre, et plus encore selon laquelle le son composé du Klang est conditionné par une localité sonore dans une même couleur auditive, où les sons « sonnent l'un dans l'autre ». Sans cette dimension spatiale (räumiche Breite), nous n'aurions pas de G estalt reconnaissable au plan harmonique ${ }^{34}$. L'inversion des catégories musicales, telle que la propose Ehrenfels, est complète. La « relation d'analogie » entre $\mathrm{G}$ estalten nous renseigne d'abord sur la nature du complexe. Ensuite, il faut bien constater qu'Ehrenfels retire aux « éléments » une partie de leur réalité objective. En donnant à la G estalt une primauté ontologique d'ordre supérieur, c'est la notion même de connexion qu'il dénoue. Parce que ces liaisons entre éléments n'ont rien d'exclusif et se multiplient indéfiniment, la qualité «formatrice » de la G estalt se voit dotée d'une sorte d'élasticité : Ehrenfels passe alors des espèces du tempo musical et de la variation tonale à la contemplation des surfaces peintes, puis à la participation du spectateur aux phénomènes de la danse. Sans doute, $M$ ach reliait déjà la génération des formes aux motifs décoratifs et ornementaux qui plaisent à l'œil ${ }^{35}$. M ais il a stipulé avec plus de rigueur qu'Ehrenfels en quoi le Klang est intrinsèquement un son composé, et pourquoi cette composition qui lui appartient en propre reste « contingente ». À l'inverse, le plaisir pris à la consonance qui est de loin le plus discuté par les acousticiens naturalistes - repose sur une fusion (Verschmelzung) très spéciale, d'un autre genre que celle d'une G estalt spatialisée ${ }^{36}$. Stumpf, on le sait, en a fait le concept essentiel de sa théorie. Pour $M$ ach, la perception des phrases mélodiques active cette fusion sur le trajet d'un ensemble de terminaisons nerveuses associées à la vibration des osselets. II hésite néanmoins à justifier cette réduction dans le passageclef que cite Ehrenfels, en en coupant la dernière phrase:

Si deux suites de sons prennent pour point de départ deux notes différentes, en progressant d'après les mêmes rapports de fréquences, nous reconnaissons par la sensation tout aussi immédiatement dans les deux cas la même mélodie que, dans deux dessins géométriquement semblables et disposés de manière semblable, nous reconnaissons une $G$ estalt identique. D es mélodies identiques, situées

32. Ehrenfels, « Uber Gestaltqualitäten », p. 22-23.

33. Ibid., p. 24.

34. Ibid., p. 24.

35. On trouve aussi chez M ach l'idée que nous pouvons « fixer » sur la partition, selon l'angle de vue spatial ou harmonique que nous choisissons, une impression déterminée, comme pour un ornement dont l'impression varierait selon les $G$ estalten optiques que nous reconnaissons ( $D$ ie A nalyse der Empfindungen, Ch. XIII, p. 12).

36. Pour lui, la perception des phrases mélodiques active cette fusion sur le trajet d'un ensemble de terminaisons nerveuses : le concept de fusion est déjà discuté par $\mathrm{H}$ ering avant Stumpf de manière distincte, mais on ne peut expliquer ici cette différence importante. 
dans des registres différents, peuvent être désignées comme ayant une Tongestalt identique ou comme des figures mélodiques [Tongebilde] semblables ${ }^{37}$.

Cette citation, qui a beaucoup frappé Ehrenfels, montre bien cependant que le terme de G estalt est encore ambivalent en ce qui concerne la structure mélodique (le dessin) de la phrase musicale. L'identité est, pour $M$ ach, une fonction de la ressemblance : un fait logique, plutôt émotionnel. Ainsi le résultat « psychologique » de la recognition est-il conditionné par le fait logique de l'identité. Toutefois, il ne fait pas de doute que c'est à partir de là qu'Ehrenfels est conduit à l'extrapolation suivant laquelle nous remplirions les « contenus » de l'intervalle en fonction de la G estalt elle-même, ainsi qu'il l'indique dans une parenthèse, puis à la fin de son article ${ }^{38}$. Cette inférence est a priori très douteuse : elle implique que la connexion (liant entre eux des sons indépendants d'elle) puisse être détachée mentalement du rapport physique des fréquences, en plus de se révéler par elle-même productrice de ce « contenu positif » dont il parlait. M ais Ehrenfels n'en a cure, et il est convaincu que la liaison que nous effectuons est totalement «psychique $\gg .{ }^{39}$

La saisie dont il parle n'est possible, au contraire, que sur le fond d'un continuum mélodique qui serait déjà entièrement qualitatif, sans plus d'égard particulier dans ce cas pour ce qu'il désigne commele « fondement » relatif de la qualité (les rapports de fréquence). Les dernières lignes de son texte vont jusqu'à imaginer des U rqualitäten, aussi primitives que les éléments eux-mêmes, et des formes de divisions chromatiques dans le son pur qui semblent beaucoup plus proches des conceptions de Brentano. Comme son propos reste centré sur la capacité d'identifier une mélodie donnée, et de définir l'expérience auditive de cette nature par une sorte de fusion mélodique s'effectuant au premier plan des conditions de la discrimination sonore, il nous faut donc conclure, sur cet aspect du moins, qu'Ehrenfels s'est inspiré

37. I bid., p. 232 (trad. fr. p. 251).

38. Ehrenfels, « Uber G estaltqualitäten », p.19 et 38.

39. $M$ ach signale bien, en effet, qu'il y a une « coloration » tonale de la phrase musicale (Färbung), mais, comme ce ne sont pas directement des rapports de fréquence qui sont « reconnus » par l'oreille, il doit préserver une identification claire de l'intervalle à partir du stimulus oscillatoire et de lui seul. Sa théorie des « colorations supplémentaires » permet, en même temps que l'élimination des battements - source du plaisir que nous prenons à la consonance - de qualifier ensuite le type de liaison que l'on a dans une sensation de tierce, et de maintenir vigoureusement que deux tierces « qualitativement semblables » ne sont pas semblables par le simple fait qu'elles ont la même intensité physique, mais parce que les deux colorations se rencontrent « occasionnellement » sur un seul nerf, c'est-à-dire du fait même que cette rencontre ne s'explique pas seulement physiquement. La qualité résultant de la liaison produite dans le G ebilde, la manière dont "ça sonne», dirait le musicien, échappe à la contingence propre de la couleur sonore en fonction du genre d'association réciproque, ou de dépendance mutuelle, qu'entretiennent les sensations de tierces, et sans plus supposer alors la « fusion » harmonique que défendait Stumpf à l'encontre du dessin mélodique. 
du travail thématique du matériau musical des post-wagnériens et de Bruckner en particulier, en y ajoutant l'affinité entre couleurs visibles et couleurs sonores, dont l'origine « analogique » se trouve chez $\mathrm{M} \mathrm{ach}^{40}$.

En résumé, la forme de la connexion pourrait n'être pas analytiquement nécessaire à l'établissement du complexe : ce second trait donnera lieu aux reconstructions de $M$ einong, puis à la mise en place précise que fournira Köhler en 1920, en insistant sur la qualité psychologique du tout «indécomposable » de la G estalt.

\section{4}

L'importance de la découverte d'Ehrenfels est ailleurs, et repose surtout dans la tentative de systématisation des divers ordres de $\mathrm{G}$ estalt. Sa définition ne confond en aucun cas les qualités de $\mathrm{G}$ estalt avec les complexes qui les soutiennent. Tel est sans conteste son aspect le plus remarquable. Aussi faut-il rappeler encore sa définition :

Par le terme de qualités de $G$ estalt, nous entendons un certain contenu positif de représentation qui est lié dans la conscience à la présence de complexes représentatifs, lesquels consistent en éléments mutuellement séparables ( $c$ 'està-dire représentables indépendamment les uns des autres). Chaque complexe représentatif qui est nécessaire à la mise en présence des qualités de $\mathrm{G}$ estalt, nous l'appelons fondement ( $G$ rundlage) de cette qualitét ${ }^{41}$.

En d'autres termes, on peut remplacer la notion de « dépendance » par la relation de « fondation » entre les éléments primaires de l'expérience perceptive. La G estalt est un tout particulier, découvre le premier Ehrenfels : elle est qualitativement inanalysable en parties, en dépit du soubassement de ses éléments constituants. Smith et M ulligan font justement observer à ce sujet ${ }^{42}$ qu'Ehrenfels imagine d'abord une «fondation ontologique réciproque » entre les qualités et les complexes élémentaires, puis qu'il écarterait cette hypothèse dans la mesure où certaines $G$ estalten peuvent servir de fondements à d'autres. Ehrenfels reviendrait alors à l'idée d'une dépendance unilatérale de la $G$ estalt par rapport au complexe amorphe des éléments. Dans $L$ 'A nalyse des sensations, $M$ ach défendrait plutôt une dépendance mutuelle : il n'aurait pas réussi à penser l'autosuffisance des complexes. Ehrenfels profite des faiblesses terminologiques de son aîné à propos du fait de savoir quelle sorte de liaison intime appartient proprement à l'objet composite de la Gestalt. Pour $M$ ach, elle ne se distingue pas des autres formes de relations qu'il s'applique à découvrir entre des sensations proprioceptives. Quoique celles-ci ne donnent jamais lieu à la formation d'une G estalt « visuelle», des

40. M ach, D ie A nalyse der Empfindungen, ch. XIII, p. 11-12.

41. Ehrenfels, « Uber Gestaltqualitäten », p. 21.

42. M ulligan, $K$ et B. Smith, «M ach and Ehrenfels: The Foundations of Gestalt Theory », p. 132. 
représentations diffuses leur sont associées de façon indubitable (par exemple dans les cas de vertige ou d'agnosie tactile). Pourtant, nous I'avons montré ci-dessus ${ }^{43}$, cette « forme » de la complexion, qui donne son nom même à l'entité de la G estalt, ne s'émancipe pas franchement des connexions purement physiologiques qui s'effectuent dans le tissu neurophysiologique entre divers quale nerveux, connexions au sujet desquelles $M$ ach avoue simplement ne rien savoir.

Le fait qu'il s'intéresse surtout aux sensations d'espace et aux sensations de temps (notamment pour les $\mathrm{G}$ estalten rythmiques), sans plus se préoccuper du sensorium interne issu de l'héritage kantien, reste son apport le plus original. Q uand il insiste sur la dépendance de nos représentations visà-vis des « éléments », ou sur la dépendance « mutuelle et réciproque » des éléments entre eux, le type de relation - et non pas justement l'invariant formel - qu'implique dans l'un et l'autre cas une telle dépendance le concerne bien plus que l'attribution d'une qualité substantielle surajoutée aux substrats que l'on considère. $\mathrm{O}$ I'a dit, $\mathrm{M}$ ach rejette expressis verbis toute ontologie déterminable pour ces mêmes substrats élémentaires. Ce qui explique la puissance de son relationnisme généralisé qui se fonde, en effet, sur la constance des relations connectives entre les phénomènes. $\mathrm{N}$ ous avons déjà admis aux paragraphes 2 et 3 que, chez lui, la nature de la connexion précède logiquement le style de configuration gestaltiste ou de complexion dont s'occupe la G estalt. On devine plus clairement désormais la nouveauté de l'essai d'Ehrenfels dans lequel est présupposée une relation « extrinsèque », spécifiquement psychologique, nécessaire à la perception du complexe. R evendiquant unesaisie de la $\mathrm{G}$ estalt tout à la fois simple et autonome, et qui procède de l'appréhension d'une entité radicalement nouvelle par rapport à la sommation des éléments, Ehrenfels ne critique pas seulement la présentation triviale ou contingente d'un agrégat : il remet pratiquement en cause la limitation interne du champ de variation caractéristique de toute connexion.

Le choix de présentations mélodiques « toutes faites » que critiquera sévèrement $M$ einong dans la recension qu'il fait d'Ü ber $\mathrm{G}$ estal tqualitäten comme si, dit-il, pour qui lirait naïvement son essai, I'acoustique eût été fondée dans le phénomène musical, et non l'inverse - ne fait qu'illustrer ce parti pris. La raison en est simple. La transposition harmonique d'une G estalt nous donnerait encore une «figure mélodique», mais elle ne serait plus reconnaissable analogiquement à la simple écoute, ni du même coup mémorisable. Ce qui importe au contraire pour Ehrenfels est que la qualité de forme soit attribuée au complexe par la mémoire, et en même temps qu'elle nous soit donnée avec lui par un « acte » de conscience, pourvu que des fundamenta (un peu moins mystérieux peut-être que les éléments de $M$ ach) demeurent en principe isolables en tant que tels. R etenons qu'il y parvient en superposant aux complexes proprement dits des qualités imputables à ces

43. Voir le $§ 1$. 
complexes. Ce n'est qu'ensuite qu'il procède à une extension et à une généralisation de $\mathrm{G}$ estalten d'ordre supérieur, par toutes sortes d'interpolations surprenantes, incluant même les habitus quotidiens, qui ne sont peut-être pas très éloignés des « formes de vie » dont on connaît la fortune à venir.

\section{5}

Pourtant, si nous admettions (en marquant ainsi les différences et non les rapprochements) que le problème d'Ehrenfels relève moins de la « psy-chologie génétique » que de l'esthétique sous sa forme la plus généreuse, nous n'aurions pas conduit l'évaluation jusqu'à son terme. $\mathrm{N}$ ous devons aussi admettre, avec M einong, que le « moment qualitatif », tel qu'il est présenté de manière positive dans Über $\mathrm{G}$ estaltqualitäten, pourrait favoriser le malentendu.

M einong objecte tout d'abord à Ehrenfels le fait que $G$ estalt et $Q$ ualität soient nommées I'une par l'autre, et désignées de façon circulaire, au détriment de la forme de complexion qu'elles impliquent. L'intérêt de la recension à chaud que donne M einong est d'insister sur le contenu « fondé » de la G estalt : il reproche notamment à Ehrenfels de lui avoir offert une extension lexicale abusive sans correspondance avec ce contenu. M einong écrit que si tout fondement appelle un « contenu fondé », « les représentations de ces contenus devront aussi s'appeler des représentations fondées », et

comme les fondements sur lesquels elles reposent en sont la condition, elles ne peuvent pas être autonomes vis-à-vis de ces dernières : des représentations non fondées peuvent aussi servir à les renforcer, en tant qu'elles sont autonomes précisément, de telle sorte que des représentations à la fois fondées et autonomes produisent dans le sens que nous venons de préciser une complète disjonction. ${ }^{44}$

M einong poursuit en indiquant que la confrontation entre cequi est au fondement de quelque chose et ce qui est fondé est le paradigme logique de toute relation. II importe ainsi de comprendre, sel on lui, que tout fait relatif (quelconque) est par nature un fait complexe :

il n'y a pas de relation sans complexion, mais il n'y a pas non plus de complexion dont les parties constituantes ne soient pas au moins en relation entre elles et avec la complexité du tout dans la mesure où elles sont effectivement des parties de ce tout. ${ }^{45}$

Ce qui permet d'éviter le malentendu est le fait de savoir à quel niveau se situel'examen psychologique des composés psychiques par rapport à ceux que nous considérons comme réels. La couleur apparaît, par exemple, à tout

44. M einong, Alexis, Zur Psychologie der Komplexionen und Relationen, A bhandlung V, 1891, p. 289-329.

45. Ibid. 
instant en relation complexe avec l'étendue, et chaque détermination de lieu n'en est pas moins en relation continue avec l'environnement local. L'erreur psychologique, insiste $M$ einong, provient de ce que nous croyons qu'il est nécessaire de faire appel à la conscience réflexive pour représenter des complexes et des relations. L'idée de la couleur et celle de l'étendue auxquelles nous pensons dans leur rapport de dépendance sont bien autre chose que des représentations de couleur et des représentations d'étendue associées dans une même forme. D ressant un réquisitoire assez sévère contre l'essai, I'auteur de la recension conteste son examen des relations de comparabilité et de compatibilité, qu'Ehrenfels avaient assimilées à des $\mathrm{G}$ estalten de second ordre où les fondements élémentaires devenaient compatibles et non contradictoires dans leurs contenus mêmes, à partir de la relation qualitative invoquée. Ce n'est là rien qu'un « brouillage » de la terminologie du fondement, lui reproche $M$ einong.

II est vrai que, selon Ehrenfels, le « contenu fondé » ne coïncide pas avec la relation de complexité qui le soutient : les remarques novatrices et hardies qu'il apporte dans le domaine des formes et des mélodies semblent se rapporter au complexe et à lui seul, sans statuer sur la nature de la relation fondatrice. $M$ ais tout cela prouve en réalité, de l'avis de $M$ einong, « que la complexion pourrait éventuellement se substituer à la relation pour être confrontée directement aux fondements ». Cette confrontation prend la forme d'une comparaison toute simple, entendue au sens littéral. Sur ce point, le jugement de $M$ einong se voudrait sans appel :

Du fait quel'analyse se consacre aux contenus fondés, il lui échappe ce qu'elle cherche. II en va tout autrement quand on compare: si quelqu'un trouve semblables le rouge et l'orange, pour lui la ressemblance sera certainement reliée de quelque manière au rouge et à l'orange, mais le rapport [entre eux] est notablement plus serré, ainsi n'est-il pas difficile de confronter la ressemblance et les contenus comparés, tandis qu'il n'existe aucun mot qui exprime à la fois le rouge, l'orange et leur ressemblance. ${ }^{46}$

II ressort de cette condamnation que le propos d'Ehrenfels est largement sous-estimé par $M$ einong, qui sera contraint plus tard de revenir sur certaines de ses idées avec plus d'indulgence. II ne reviendra pas néanmoins sur sa récusation de l'option musicale présentée ci-dessus. Pour $M$ einong, ni le référent chromatique du Klang et encore moins la succession mélodique des couleurs sonores ne pourront jamais offrir le modèle « réel » del'idéalité du complexe. La dispute sur la consistance ou sur l'intégrité du « tout » ontologique de la $\mathrm{G}$ estalt venait de s'ouvrir. Elle quittait le terrain du type de connexion entre éléments pour celui de la forme de complexion dans la représentation.

46. I bid., p 295. 